\title{
Minimisation and Parameter Estimation in Image Restoration Variational Models with $\ell_{1}$-Constraints
}

\author{
M. Tao* \\ Department of Mathematics, Nanjing University, Nanjing, 210093, China. \\ Received 21 January 2017; Accepted (in revised version) 6 August 2017.
}

\begin{abstract}
Minimisation of the total variation regularisation for linear operators under $\ell_{1}$-constraints applied to image restoration is considered, and relationships between the Lagrange multiplier for a constrained model and the regularisation parameter in an unconstrained model are established. A constrained $\ell_{1}$-problem reformulated as a separable convex problem is solved by the alternating direction method of multipliers that includes two sequences, converging to a restored image and the "optimal" regularisation parameter. This allows blurry images to be recovered, with a simultaneous estimation of the regularisation parameter. The noise level parameter is estimated, and numerical experiments illustrate the efficiency of the new approach.
\end{abstract}

AMS subject classifications: 68U10, 65J22, 65K10, 90C25

Key words: Parameter selection, $\ell_{1}$-constraints, alternating direction method of multipliers, impulsive noise, image processing.

\section{Introduction}

The restoration of images is a challenging problem, and here images $\bar{x} \in \mathbb{R}^{n}$ corrupted by impulse noise and blurring effects are discussed. Blurring issues are almost unavoidable in contemporary imaging systems, and corruption by impulse noise emerges from bit errors in transmission, wrong pixels and faulty memory locations in hardware $[1,6,12,23]$. In the model, an observed image $f$ is represented by the equation

$$
f=N(K \bar{x}),
$$

where $N$ and $K$ denote the impulse noise and blurring effect, respectively. In applications, there are two main impulse noise sources - viz. salt-and-pepper and random-valued impulse noise [9]. Image restoration problems are usually ill conditioned and the direct solution of the system (1.1) rarely produces satisfactory results. To address this problem, one can use a regularisation procedure - e.g. the total variational (TV) regularisation [35], a

${ }^{*}$ Corresponding author. Email address: taom@nju.edu.cn (M. Tao) 
wavelet transform [8], or a nonlocal regularisation [21]. The corresponding unconstrained model for the image deblurring can be written in the form

$$
\min _{x \in \mathbb{R}^{n}}\left\{\|D x\|+\lambda \Phi_{\text {fit }}(x, f)\right\},
$$

where $D$ is a linear operator and $\Phi_{\text {fit }}(x, f)$ is the data-fitting term. In particular, if $D$ is the discrete gradient operator $\nabla$, then this model (1.2) represents the TV regularisation where the parameter $\lambda$ balances the data fidelity with the regularity. The constrained counterpart of the model (1.2) is

$$
\begin{array}{cl}
\min _{x \in \mathbb{R}^{n}} & \|D x\| \\
\text { s.t. } & \Phi_{\text {fit }}(x, f) \leq \tau
\end{array}
$$

with $\tau$ representing the noise level, and constrained models have been also used in image recovery $[32,41,45]$. For the impulse noise, one sets $\Phi_{\text {fit }}(x, f)=\|K x-f\|_{1}$ in either of (1.2) or (1.3), which are respectively called unconstrained and constrained $\ell_{1}$-models.

Numerical methods have been used to solve the unconstrained model (1.2) under the TV regularisation [17, 40,45], a wavelet regulariser [14], and a nonlocal regularisation [15]. The associated minimiser $\hat{x}=\hat{x}(\lambda)$, and hence the recovered image, depends on the choice of $\lambda$. Usually, the parameter $\lambda$ is determined manually by a trial-and-error method, but several techniques have been developed to detect the best parameter $\lambda$ automatically - e.g. the L-curve criterion [24], the generalised cross-validation (GCV) $[19,20]$, the normalised cumulative or residual periodogram approach [25,36], variational Bayes approaches [2,3,34], and Morozov's discrepancy principle (MDP) [31]. On the other hand, constrained $\ell_{1}$-models have only recently been considered, and if a priori information about noise is available then the constrained model is more attractive [41,47].

To solve unconstrained $\ell_{1}$-models, various algorithms have been developed recently. In particular, a fast TV deblurring algorithm (FTVd) combines the variable splitting and quadratic penalised technique [40]. Each subproblem can be solved by either shrinkage or the fast Fourier transform (FFT), so the FTVd method performs much better than many other methods $[17,42]$. An incremental version of the FTVd approach involving the alternating direction method of multipliers (ADMM) can be used to solve an unconstrained $\mathrm{TV}-\ell_{1}$-model. Wu et al. [44] developed the inexact augmented Lagrangian method equivalent to the ADMM. All of these approaches assume that a suitable regularisation parameter in the unconstrained model is known - and it can be determined manually by a trial-anderror method, but this procedure is often very slow, so one may prefer to restore images corrupted by impulse noise via the constrained TV- $\ell_{1}$-model. Weiss et al. [45] used Nesterov's first-order scheme to do so, and this approach was improved by $\mathrm{Ng}$ et al. [33], but it requires inner iterations to describe projections onto an $\ell_{1}$-ball.

The parameter $\lambda$ in the unconstrained model (1.2) can also be determined by some classical methods - e.g. if the regularisation term has a quadratic form the GCV evaluation formula may be employed. However, this formula cannot be used directly in the seminormbased model (1.2) due to the non-linearity of the seminorms $\|D \cdot\|_{2}$. The regularisation parameter can be estimated by quadratic approximation of the seminorm term [30], or by 
using an extra quadratic penalty term instead of the non-smooth term, but the resulting model involves an additional parameter, under-smoothes the solution and can lead to multiple minimisers for the GCV function [28]; and the L-curve method [24, 29] for finding the regularisation parameter is expensive and the location of the L-curves is difficult. Another popular method to evaluate the regularisation parameter is the MDP, which selects the optimal regularisation parameter $\lambda$ by matching the norm of the residual (the violation of the data-fitting term) to an upper bound. This means that the solution $x(\hat{\lambda})$ satisfies the discrepancy equation

$$
\Phi_{\text {fit }}(\hat{x}(\lambda), f)=\tau .
$$

Recall that $\hat{x}(\lambda)$ is the minimiser of (1.2), and note that parameter-selection methods for Gaussian noise [47] and Poisson data [8, 41] based on the MDP allow the discrepancy equation to be solved iteratively. These approaches cannot be used for the evaluation of the regularisation parameter in unconstrained $\ell_{1}$-models, however.

The goal of this work is to provide a fast scheme for simultaneous solution of constrained $\ell_{1}$-models, and to propose a method for the evaluation of the regularisation parameter in the unconstrained model (1.2). The TV regulariser $\|\nabla x\|$ in the model (1.2) is isotropic if the $l_{2}$-norm is used, and anisotropic for the $l_{1}$-norm. Our approach is applicable in both isotropic and anisotropic TV regulariser. Here the TV regulariser for the models (1.2) and (1.3) in the isotropic case $\|\cdot\|:=\|\cdot\|_{2}$ is considered, for the treatment of the anisotropic case is similar. Moreover, our approach also works for tight frames and nonlocal regularisers, and allows for more general data-fitting terms - e.g. for the $\ell_{\infty}$-norm, suitable for uniform noise [46], and the norm $\|S K x-f\|_{1}$ where $S$ is a mask matrix used in image inpainting. Let us reformulate (1.3) as a separable convex problem, and apply the alternating direction method of multiplier method (ADMM) [18,22]. This differs from reformulations in Refs. [33,45], and provides a closed-form solution to each subproblem, simultaneously introducing a scalar sequence converging to the "optimal" regularisation parameter $\lambda$ in (1.2). The recovered quality of the unconstrained model with such an "optimal" $\lambda$ is comparable to the constrained model. Moreover, the ADMM can be replaced by other first-order algorithms. Theoretical analysis based on Lagrange dual theory shows the interconnection between the constrained and unconstrained models, and the high efficiency of our approach. A new method to evaluate the parameter $\tau$ in the model (1.3) is now proposed.

Throughout, the following notation is used. Let $x=\left(x_{1}, x_{2}, \cdots, x_{n}\right)^{\top}$ be extended by periodic boundary conditions. Only square images such that $n=m^{2}, m \in \mathbb{N}$ are considered. The operator $\nabla_{i} x \in \mathbb{R}^{2}$ represents the first-order finite difference of $x$ at the pixel $i$ in each of the horizontal and vertical directions - i.e.

$$
\nabla_{i} x:=\left(\left(\nabla^{(1)} x\right)_{i},\left(\nabla^{(2)} x\right)_{i}\right)^{\top} \in \mathbb{R}^{2}, \quad i=1, \cdots, n,
$$


where

$$
\begin{aligned}
\left(\nabla^{(1)} x\right)_{i} & :=\left\{\begin{array}{l}
x_{i+m}-x_{i} \text { if } 1 \leq i \leq m(m-1) \\
x_{\bmod (i, m)}-x_{i} \text { otherwise }
\end{array}\right. \\
\left(\nabla^{(2)} x\right)_{i} & :=\left\{\begin{array}{l}
x_{i+1}-x_{i} \text { if } \bmod (i, m) \neq 0 \\
x_{i-m+1}-x_{i} \text { otherwise }
\end{array}\right.
\end{aligned}
$$

The discrete gradient operators $\nabla^{(1)}$ and $\nabla^{(2)}$ are $n \times n$ matrices, where the $i$-th rows of $\nabla^{(1)}$ and $\nabla^{(2)}$ respectively correspond to the first and second rows of $\nabla_{i}$. The discrete total variation seminorm is $\|\nabla x\|=\sum_{i=1}^{n}\left\|\nabla_{i} x\right\|_{2}$, where the quantity $\left\|\nabla_{i} x\right\|_{2}$ measures the total variation of $x$ at the pixel $i$. The resulting total variation is called isotropic. (On the other hand, the corresponding total variation is referred to as anisotropic when the $l_{1}$-norm is used, but here the symbol $\|\cdot\|$ is reserved for the $l_{2}$-norm.) We also let $\nabla \equiv\left(\nabla^{(1)} ; \nabla^{(2)}\right) \in$ $\mathbb{R}^{2 n \times n}$ be the global first-order finite-difference operator such that $\nabla^{\top} \nabla=\sum_{i=1}^{n} \nabla_{i}^{\top} \nabla_{i}$, and denote the range and null spaces of an operator $A$ by $\mathscr{R}(A)$ and $\mathscr{N}(A)$, respectively. A diagonal matrix $S$ is called a mask matrix if its diagonal entries are 1 or 0 , where the values 1 and 0 correspond to sampled and missing pixels, respectively. Given a vector $x$ and an index set $\Omega \subseteq\{1, \cdots, n\}$, the symbol $\Omega^{C}$ denotes the complement of $\Omega$ in $\{1, \cdots, n\}, x_{\Omega}$ denotes the entries of the vector $x$ restricted on the set $\Omega$, and $\stackrel{\circ}{D}$ the relative interior of the set $D[4]$.

In Section 2, MDP-based approaches for parameter selection are discussed, but shown to be inapplicable in $\ell_{1}$-models. Section 3 establishes relationships between the constrained and unconstrained models. In Section 4, an ADMM-based approach is used to solve the constrained $\ell_{1}$-type model (1.3), and a sequence of the Lagrange multipliers is shown to converge to the "optimal" regularisation parameter. The numerical results presented in Section 6 confirm the validity of the theoretical analysis. Concluding remarks are in Section 7, and the Appendix contains the solution of an image inpainting problem.

\section{Parameter Selection by MDP-based methods}

In order to find the parameter $\lambda$ by an MDP-based method, one has to find the solution of the discrepancy equation (1.4). Wen \& Chan [47] developed a proximal point method using a representation of the TV term in the dual formulation, and similar MDP-based approaches for Poisson noise and multiplicative Gamma noise have been used $[8,41]$ where first-order primal-dual algorithms to solve the constrained model have been considered. One of the least squares constrained problems studied is

$$
\min _{x \in \mathbb{R}^{n}} \frac{1}{2}\|x-q\|^{2} \quad \text { subject to } \quad \Phi_{\text {fit }}(x, f) \leq \tau,
$$

where $q \in \mathbb{R}^{n}$ is a given vector. The corresponding unconstrained problem

$$
\min _{x \in \mathbb{R}^{n}}\left\{\frac{1}{2}\|x-q\|^{2}+\lambda \Phi_{\text {fit }}(x, f)\right\}
$$


has an analytical solution for the Gaussian noise data-fitting term $\Phi_{\mathrm{fit}}(x, f)=\|K x-f\|^{2}$, and for the Kullback-Leibler divergence distance

$$
\Phi_{\mathrm{fit}}(x, f)= \begin{cases}\langle 1, f \log (f)-f \log (K x)+K x-f\rangle & \text { if }(K x)>0 \\ \infty & \text { otherwise }\end{cases}
$$

in the case of Poisson or multiplicative Gamma noise. If $\hat{x}(q, \lambda)$ is the corresponding solution of (2.1), then the discrepancy equation

$$
\Phi_{\text {fit }}(\hat{x}(q, \lambda), f)=\tau
$$

has a unique solution since the function $\Phi_{\text {fit }}(\hat{x}(q, \lambda), f)$ is strictly monotone with respect to $\lambda$. Thus such methods produce two sequences $\hat{x}(q, \lambda)$ and $\lambda$, where the first converges to the solution of the constrained model and the second to the regularisation parameter of the unconstrained model. It is notable that the applicability of the above approach depends on both the exact analytical solution of the problem (2.1) and the strict monotonicity of the function $\Phi_{\text {fit }}(\hat{x}(q, \lambda), f)$ - and these two conditions are satisfied for Gaussian, Poisson and multiplicative Gamma noise. For unconstrained $\ell_{1}$-models, the first condition can be satisfied by replacing the term $K x$ with a new variable such that the problem (2.1) still has a closed-form solution $[8,41]$. On the other hand, the function $\Phi_{\text {fit }}(\hat{x}(q, \lambda), f)$ is not strict monotone with respect to $\lambda$ and the discrepancy equation can have multiple solutions. Thus these approaches in Refs. $[8,41,47]$ are not applicable to unconstrained $\ell_{1}$-models, and one has to find another way to find a suitable parameter $\lambda$.

\section{Unconstrained and Constrained Convex Problems}

For a convex function $F: \mathbb{R}^{n} \rightarrow \mathbb{R}$ and a proper convex lower semi-continuous function $G: \mathbb{R}^{n} \rightarrow \mathbb{R} \cup\{+\infty\}$, let us consider the minimisation problems

$$
\begin{aligned}
& \min _{x \in \mathbb{R}^{n}}\{G(x)+\lambda F(x)\}, \quad \lambda>0, \\
& \min _{x \in \mathbb{R}^{n}} G(x) \text { subject to } F(x) \leq \tau .
\end{aligned}
$$

The Lagrange function of the constrained problem (3.2) is defined as

$$
L(x, p)=G(x)+p(F(x)-\tau),
$$

where $p \geq 0$ is the Lagrange multiplier. Recall $(\hat{x}, \hat{p}) \in \mathbb{R}^{n} \times \mathbb{R}_{+}\left(\mathbb{R}_{+}:=\{x \in \mathbb{R}: x \geq 0\}\right)$ is a saddle point of the Lagrangian function (3.3) if and only if

$$
L(\hat{x}, p) \leq L(\hat{x}, \hat{p}) \leq L(x, \hat{p}),
$$

for all $p \geq 0$ and for all $x \in \mathbb{R}^{n}$. If the function (3.3) has a saddle point $(\hat{x}, \hat{p})$, then $\hat{x}$ is a solution of the problem (3.2). To obtain the reverse conclusion, let us recall Slater's 
constraint qualification (SCQ):

$$
\left\{\begin{array}{l}
\text { If } F(x) \text { is a nonlinear function, } \\
\quad \text { then there is } x_{0} \in \operatorname{dom}(G) \bigcap \operatorname{dom}(F) \bigcap \stackrel{\circ}{D} \text { where } D=\{x \mid F(x) \leq \tau\} ; \\
\text { If } F(x) \text { is a linear function, then there is } x_{0} \in \operatorname{dom}(G) \bigcap \operatorname{dom}(F) \bigcap D .
\end{array}\right.
$$

Consequently, if the problem (3.2) has a solution $\hat{x}$ and the SCQ is fulfilled, then $(\hat{x}, \hat{p})$ is a saddle point of (3.3) for some $\hat{p} \geq 0$.

The following theorem shows the relationship between constrained and unconstrained problems.

Theorem 3.1. Assume that $F: \mathbb{R}^{n} \rightarrow \mathbb{R}$ is convex and $G: \mathbb{R}^{n} \rightarrow \mathbb{R} \cup\{+\infty\}$ is proper, convex, and lower semi-continuous. If the minima

$$
\tau_{L}:=\min _{x \in \mathbb{R}^{n}} F(x) \quad \text { and } \quad \tau_{U}:=\min _{x \in \arg \min _{x \in \mathbb{R}^{n}} G(x)} F(x)
$$

exist, then $\tau_{L}<\tau_{U} \Leftrightarrow \operatorname{argmin}_{x \in \mathbb{R}^{n}} F(x) \cap \operatorname{argmin}_{x \in \mathbb{R}^{n}} G(x)=\emptyset$.

Let

$$
\tau_{L}<\tau<\tau_{U}
$$

i) If $\hat{x}$ is a solution of (3.2), then $F(\hat{x})=\tau$.

ii) Assume that the SCQ (3.5) is satisfied. If $\hat{x}$ is a solution of (3.2) and $(\hat{x}, \hat{p})$ is a saddle point of the Lagrangian (3.4), then $\hat{p}>0$. Moreover, $\hat{x}$ is a solution of (3.1) with $\lambda=\hat{p}$ -i.e.

$$
\hat{x} \in \underset{x \in \mathbb{R}^{n}}{\operatorname{argmin}}\{G(x)+\hat{p} F(x)\} .
$$

Proof. It follows from the definition of $\tau_{L}$ and $\tau_{U}$ that $\tau_{L} \leq \tau_{U}$. Moreover,

$$
\tau_{L}=\tau_{U} \Leftrightarrow \arg \min _{x \in \mathbb{R}^{n}} F(x) \cap \arg \min _{x \in \mathbb{R}^{n}} G(x) \neq \emptyset \text {, hence } \tau_{L}<\tau_{U} .
$$

i) Let $\tau_{L}<\tau<\tau_{U}$, to proceed to show that any solution $\hat{x}$ of (3.2) satisfies the equation $F(x)=\tau$. First, it is notable that $\hat{x}$ is not a minimiser of $F(x)$ because $\tau>\tau_{L}$. Assume that $\hat{x}$ is a minimiser of $G(x)$. Then

$$
F(\hat{x}) \leq \tau<\tau_{U}=\min _{x \in \arg \min G(x)} F(x) \leq F(\hat{x}),
$$

which is a contradiction. Finally, the assumption $F(\hat{x})<\tau$ implies the false conclusion that $\hat{x}$ is a minimiser of $G(x)$, hence our assumption is wrong and $F(\hat{x})=\tau$.

ii) Let $\hat{x}$ be a solution of (3.2) and $(\hat{x}, \hat{p})$ be a saddle point of the Lagrangian. Assuming that $\hat{p}=0$, one obtains

$$
\hat{x} \in \arg \min _{x \in \mathbb{R}^{n}} L(x, \hat{p})=\arg \min _{x \in \mathbb{R}^{n}} G(x),
$$


so that $\hat{x}$ is a minimiser of $G(x)$ and this contradicts the condition $\tau<\tau_{U}$. Hence, $\hat{p}>0$. Finally, from the saddle point definition one has

$$
\hat{x} \in \arg \min _{x \in \mathbb{R}^{n}} L(x, \hat{p})=\arg \min _{x \in \mathbb{R}^{n}}\{G(x)+\hat{p}(F(x)-\tau)\}=\arg \min _{x \in \mathbb{R}^{n}}\{G(x)+\hat{p} F(x)\},
$$

so $\hat{x}$ is a solution of (3.1), which completes the proof.

Let us now consider the models

$$
\begin{aligned}
& \min _{x \in \mathbb{R}^{n}}\left\{\|D x\|+\lambda\|K x-f\|_{1}\right\}, \quad \lambda>0, \\
& \min _{x \in \mathbb{R}^{n}}\|D x\| \quad \text { subject to }\|K x-f\|_{1} \leq \tau, \quad \tau>0,
\end{aligned}
$$

where $K \in \mathbb{R}^{s, n}, D \in \mathbb{R}^{t, n}, f \in \mathbb{R}^{s}$ and $\|\cdot\|$ is a norm on $\mathbb{R}^{s}$. Let us also assume that

$$
\mathscr{N}(K) \cap \mathscr{N}(D)=\{0\} .
$$

The function $\|K x-f\|_{1}$ is coercive on any nontrivial subspace $Y$ of $\mathbb{R}^{n}$ with $\mathscr{N}(K) \cap Y=\{0\}$, hence the minima

$$
\tau_{L}:=\min _{x \in \mathbb{R}^{n}}\|K x-f\|_{1}, \quad \tau_{U}:=\min _{x \in \mathcal{N}(D)}\|K x-f\|_{1}
$$

exist. Recalling that the zero space of the operator $D:=\nabla$ is $\mathscr{N}(\nabla)=\left\{\alpha 1_{n}: \alpha \in \mathbb{R}\right\}$ and the blur operator $K$ usually possesses the property $K 1_{n}=1_{n}$, one has

$$
\tau_{U}:=\min _{\alpha \in \mathbb{R}}\left\|\alpha 1_{n}-f\right\|_{1},
$$

which is just the median of $f-$ cf. Ref. [37]. Let us now assume that $\tau_{L}<\tau_{U}$. The following theorem provides solvability conditions for the models (3.7) and (3.8).

Theorem 3.2. If $\mathscr{N}(K) \cap \mathscr{N}(D)=\{0\}$, then:

i) the problem (3.7) has a minimiser;

ii) and if in addition $\tau \geq \tau_{L}$, the problem (3.8) has a minimiser.

Proof. Since all norms on $\mathbb{R}^{n}$ are equivalent, the theorem is valid for any norm used in the data and regularity terms. The proof follows standard arguments.

i) It suffices to show that the objective function $E(x):=\|D x\|+\lambda\|K x-f\|_{1}$ is coercive i.e. that $E(x) \rightarrow+\infty$ as $\|x\| \rightarrow+\infty$. Assume this is not true. Then there is a sequence $\left\{x^{k}\right\} \in \mathbb{R}^{n}$ such that $\lim _{k \rightarrow \infty}\left\|x^{k}\right\|=\infty$ but

$$
\left\|D x^{k}\right\|+\lambda\left\|K x^{k}-f\right\|_{1} \leq C,
$$

and consequently

$$
\left\|D x^{k}\right\| \leq C, \quad\left\|K x^{k}-f\right\|_{1} \leq C,
$$

for any $k \in \mathbb{N}$. Let $\mathscr{N}(D) \oplus \mathscr{R}\left(D^{\top}\right)$ be the orthogonal decomposition of $\mathbb{R}^{n}$ and let $x^{k}=$ $x_{0}^{k}+x_{1}^{k}$ be the corresponding representation of an element $x^{k}$. The operator $D$ considered 
on the space $\mathscr{R}\left(D^{\top}\right)$ is injective. Hence for the element $D x^{k}=y^{k}$ one has $x_{1}^{k}=D^{\dagger} y^{k}$, where $D^{\dagger}$ is a Moore-Penrose inverse of $D[5]$; and using (3.11) one obtains

$$
\left\|x_{1}^{k}\right\|=\left\|x^{k}-x_{0}^{k}\right\|=\left\|D^{\dagger} y^{k}\right\| \leq\left\|D^{\dagger}\right\|\left\|y^{k}\right\|=\left\|D^{\dagger}\right\|\left\|D x^{k}\right\| \leq C_{2} .
$$

Moreover, the triangle inequality and (3.11) imply that

$$
\begin{aligned}
\left\|K x_{0}^{k}\right\|_{1} & \leq\left\|K\left(x^{k}-x_{0}^{k}\right)\right\|_{1}+\|f\|_{1}+\left\|K x^{k}-f\right\|_{1} \\
& \leq C_{3}\left\|x^{k}-x_{0}^{k}\right\|+\|f\|_{1}+\left\|K x^{k}-f\right\|_{1} \leq C_{4} .
\end{aligned}
$$

Let us assume there is a subsequence $x_{0}^{k_{j}}$ of $x_{0}^{k}$ such that $\left\|x_{0}^{k_{j}}\right\| \rightarrow \infty$ as $j \rightarrow \infty$. Since $\mathscr{N}(K) \cap \mathscr{N}(D)=\{0\}$, the operator $K$ is injective on $\mathscr{N}(D)$ such that the norms $\left\|K x_{0}^{k_{j}}\right\|_{1}$ tend to $\infty$, which contradicts the inequality (3.13). Thus $\left\|x_{0}^{k}\right\| \leq C_{5}$ for all $k-$ and from (3.12) the sequence $\left\{x^{k}\right\}$ is bounded, contrary to our assumption.

ii) Since $\tau \geq \tau_{L}$, the set

$$
X:=\left\{x \in \mathbb{R}^{n}:\|K x-f\|_{1} \leq \tau\right\}
$$

is nonempty. Moreover, it is closed and convex. The directions of the recession of $X$ and the objective function in (3.8) are respectively $\mathscr{N}(K)$ and $\mathscr{N}(D)$. Since $\mathscr{N}(K) \cap \mathscr{N}(D)=\{0\}$, it follows from Proposition 2.3.2 in Ref. [4] that the solution set of (3.8) is nonempty and compact.

Corollary 3.1. If $\mathscr{N}(K) \cap \mathscr{N}(D)=\{0\}$ and $\tau_{L}<\tau<\tau_{U}$, the problem (3.8) has a positive Lagrange multiplier.

The proof of this result follows from the fact that the Slater condition for the problem (3.8) is fulfilled if $\tau \geq \tau_{L}$.

\section{ADMM Approach to Constrained $\ell_{1}$-Models}

In this section, the constrained problem (3.8) is solved with the simultaneous evaluation of the regularisation parameter of the unconstrained model (3.7). More precisely, consider the model (3.8) with the TV regularisation - i.e. when $\|D x\|=\sum_{i}\left\|\nabla_{i} x\right\|$ and the blur operator $K \in \mathbb{R}^{n \times n}$. The resulting models (3.7) and (3.8) are called unconstrained and constrained TV- $\ell_{1}$-models, respectively. In passing, it is notable that our approach is also applicable to other regularisations.

The choice of the parameter $\tau$ in the constrained model (3.8) satisfies the condition (3.6). From Theorem 3.1(i), the constraint in (3.8) is tight. Assuming that the parameter $\tau$ satisfies the condition (3.6), one can rewrite the constrained TV- $\ell_{1}$-model as

$$
\begin{aligned}
& \min _{x, z_{i}} \sum_{i=1}^{n}\left\|z_{i}\right\| \\
& \text { s.t. } \nabla_{i} x=z_{i} \quad: \mu_{i} \\
& \|K x-f\|_{1}=\tau \quad: \kappa
\end{aligned}
$$


where $z_{i} \in \mathbb{R}^{2}$ is an auxiliary vector, and $\mu_{i} \in \mathbb{R}^{2}$ and $\kappa \in \mathbb{R}$ are the Lagrange multipliers of the first and second equalities in the model (4.1).

Moreover, since the equation

$$
\|K x-f\|_{1}=\tau
$$

can be rewritten as

$$
-y \leq K x-f \leq y, \quad e^{\top} y=\tau,
$$

where $e=(1,1, \cdots, 1) \in \mathbb{R}^{n}$, the model (4.1) is equivalent to the minimisation problem

$$
\begin{array}{lll}
\min _{x, y, z_{i}, w_{1}, w_{2}} \sum_{i=1}^{n}\left\|z_{i}\right\| & \\
\text { s.t. } & \nabla_{i} x=z_{i} & : \mu_{i} \\
& K x-f-y+w_{1}=0 & : v \\
& K x-f+y-w_{2}=0 & : \delta \\
& e^{\top} y=\tau & : \kappa \\
& w_{1}, w_{2} \geq 0 &
\end{array}
$$

with the Lagrange multipliers $\mu_{i} \in \mathbb{R}^{2}, v \in \mathbb{R}^{n}, \delta \in \mathbb{R}^{n}$ and $\kappa \in \mathbb{R}$. Similar to the definitions of $\left(\nabla^{(1)} x\right)_{i}$ and $\left(\nabla^{(2)} x\right)_{i}$ in Eqs. (1.5) and (1.6), consider a vector

$$
z:=\left(\begin{array}{c}
z^{(1)} \\
z^{(2)}
\end{array}\right) \in \mathbb{R}^{2 n^{2}}, \quad z_{i}:=\left(\begin{array}{c}
\left(z^{(1)}\right)_{i} \\
\left(z^{(2)}\right)_{i}
\end{array}\right) \in \mathbb{R}^{2}, \quad i=1, \cdots, n^{2} .
$$

Then the augmented Lagrangian function of the problem (4.2) is

$$
\begin{aligned}
& \mathscr{L}_{\mathscr{A}}\left(x, y, z, w_{1}, w_{2} ; \mu, v, \delta, \kappa\right) \\
= & \sum_{i=1}^{n}\left\|z_{i}\right\|+\left\langle v, K x-y-f+w_{1}\right\rangle+\frac{\beta_{1}}{2}\left\|K x-y-f+w_{1}\right\|^{2} \\
& +\left\langle\delta, K x+y-f-w_{2}\right\rangle+\frac{\beta_{1}}{2}\left\|K x+y-f-w_{2}\right\|^{2}+\left\langle\kappa, e^{\top} y-\tau\right\rangle \\
& +\frac{\beta_{2}}{2}\left\|e^{\top} y-\tau\right\|^{2}+\sum_{i=1}^{n}\left\langle\mu_{i}, \nabla_{i} x-z_{i}\right\rangle+\frac{\beta_{3}}{2} \sum_{i=1}^{n}\left\|\nabla_{i} x-z_{i}\right\|^{2},
\end{aligned}
$$

where $\beta_{1}, \beta_{2}, \beta_{3}>0$. Using the ADMM described in Refs. [18,22] starting at $x=x^{k}$, 
$y=y^{k}, \mu=\mu^{k}, v=v^{k}, \delta=\delta^{k}$ and $\kappa=\kappa^{k}$, one arrives at the following iterative scheme:

$$
\begin{aligned}
& \left(\begin{array}{c}
w_{1}^{k+1} \\
w_{2}^{k+1} \\
z^{k+1}
\end{array}\right) \leftarrow \underset{w_{1} \in \mathbb{R}_{+}^{n}, w_{2} \in \mathbb{R}_{+}^{n}, z}{\arg \mathscr{L}_{\mathscr{A}}\left(x^{k}, y^{k}, z, w_{1}, w_{2} ; \mu^{k}, v^{k}, \delta^{k}, \kappa^{k}\right),} \\
& \left(\begin{array}{c}
x^{k+1} \\
y^{k+1}
\end{array}\right) \leftarrow \arg \min _{x, y} \mathscr{L}_{\mathscr{A}}\left(x, y, z^{k+1}, w_{1}^{k+1}, w_{2}^{k+1} ; \mu^{k}, v^{k}, \delta^{k}, \kappa^{k}\right), \\
& \left(\begin{array}{c}
\mu_{i}^{k+1} \\
v^{k+1} \\
\delta^{k+1} \\
\kappa^{k+1}
\end{array}\right) \leftarrow\left(\begin{array}{c}
\mu_{i}^{k}+\beta_{3}\left(\nabla_{i} x^{k+1}-z_{i}^{k+1}\right) \\
v^{k}+\beta_{1}\left(K x^{k+1}-f-y^{k+1}+w_{1}^{k+1}\right) \\
\delta^{k}+\beta_{1}\left(K x^{k+1}-f+y^{k+1}-w_{2}^{k+1}\right) \\
\kappa^{k}+\beta_{2}\left(e^{\top} y^{k+1}-\tau\right)
\end{array}\right) .
\end{aligned}
$$

This method converges for any positive numbers $\beta_{1}, \beta_{2}, \beta_{3}[7,27]$.

Now let us split the minimisation procedure (4.3) into three independent subproblems with respect to $w_{1}, w_{2}$, and $z$. The algorithms for $w_{1}$ and $w_{2}$ are obtained by projecting the corresponding terms on the space $\mathbb{R}_{+}^{n}$ - viz.

$$
\begin{aligned}
w_{1}^{k+1} & =\mathscr{P}_{\mathbb{R}_{+}^{n}}\left[y^{k}+f-\frac{v^{k}}{\beta_{1}}-K x^{k}\right], \\
w_{2}^{k+1} & =\mathscr{P}_{\mathbb{R}_{+}^{n}}\left[y^{k}-f+\frac{\delta^{k}}{\beta_{1}}+K x^{k}\right] .
\end{aligned}
$$

The third subproblem is equivalent to $n$ two-dimensional problems of the form

$$
\min _{z_{i} \in \mathbb{R}^{2}}\left\{\left\|z_{i}\right\|+\frac{\beta_{3}}{2}\left\|z_{i}-\left(\nabla_{i} x^{k}+\frac{1}{\beta_{3}}\left(\mu^{k}\right)_{i}\right)\right\|^{2}\right\}, \quad i=1,2, \cdots, n,
$$

and it has the analytical solution

$$
z_{i}^{k+1}=\max \left\{\left\|\nabla_{i} x^{k}+\frac{1}{\beta_{3}}\left(\mu^{k}\right)_{i}\right\|-\frac{1}{\beta_{3}}, 0\right\} \frac{\nabla_{i} x^{k}+\frac{1}{\beta_{3}}\left(\mu^{k}\right)_{i}}{\left\|\nabla_{i} x^{k}+\frac{1}{\beta_{3}}\left(\mu^{k}\right)_{i}\right\|}, \quad i=1,2, \cdots, n,
$$

presented in Refs. $[39,43]$. The computational cost in solving (4.8) grows linearly with respect to $n$.

The minimisation problem (4.4) can also be simplified by splitting it into two independent subproblems, one with respect to $x$ and another with respect to $y$. The $x$ subproblem is just a least squares problem with the normal equation

$$
\left(\nabla^{\top} \nabla+2 \frac{\beta_{1}}{\beta_{3}} K^{\top} K\right) x^{k+1}=\nabla^{\top}\left(z^{k+1}-\frac{\mu^{k}}{\beta_{3}}\right)+\frac{\beta_{1}}{\beta_{3}} K^{\top}\left(2 f-w_{1}^{k+1}+w_{2}^{k+1}-\frac{v^{k}+\delta^{k}}{\beta_{1}}\right) .
$$

If the condition (3.9) holds, the coefficient matrix in Eq. (4.9) is nonsingular. Moreover, for boundary conditions periodic in $x$, the matrices $\nabla^{\top} \nabla$ and $K^{\top} K$ are block circulant 
$[10,23]$, so they are diagonalisable via the two-dimensional discrete Fourier transform (FFT) at $\mathrm{O}(n \log n)$ cost. For a symmetric blur and Neumann boundary conditions, the coefficient matrix can be diagonalised by the discrete cosine transform (DCT) at the same cost - cf. Ref. [32]. The $y$ subproblem is likewise a least squares problem with the normal equation

$$
\left(2 I+\frac{\beta_{2}}{\beta_{1}} e e^{\top}\right) y^{k+1}=\frac{\beta_{2}}{\beta_{1}} \tau e+\left(w_{1}^{k+1}+w_{2}^{k+1}\right)+\frac{v^{k}-\delta^{k}-\kappa^{k} e}{\beta_{1}} .
$$

According to the Sherman-Morrison-Woodburg theorem,

$$
\left(2 I+\frac{\beta_{2}}{\beta_{1}} e e^{\top}\right)^{-1}=\frac{1}{2} I-\frac{\frac{\beta_{2}}{\beta_{1}}}{4+2 \frac{\beta_{2}}{\beta_{1}} \cdot n} e e^{\top},
$$

and the cost of solving of the $y$-subproblem is $\mathrm{O}(n)$. Finally, the update of (4.5) for $\mu_{i}$, $v, \delta$, and $\kappa$ is straightforward and requires $\mathrm{O}(n)$ operations. Each subproblem preserves a closed-form solution, the corresponding regularisation parameter can be obtained as the limit of the sequence $\kappa^{k}$, and the per-iteration cost for the scheme (4.3)-(4.5) dominated by two FFT or two DCT operations is $\mathrm{O}(n \log n)$.

Algorithm 4.1 ADMM approach for the constrained TV- $\ell_{1}$-model (4.1).

Input $f, K, \tau>0, \beta_{1}, \beta_{2}, \beta_{3}>0$, and $\mu^{0}, v^{0}, \delta^{0}, \kappa^{0}$. Initialise $x=f, y=f$ and $\mu=\mu^{0}$, $v=v^{0}, \delta=\delta^{0}$, and $\kappa=\kappa^{0}$.

Output $\hat{x}, \hat{\kappa}$.

While "a stopping criterion is not satisfied", Do

1) Compute $w_{1}^{k+1}$ according to (4.6).

2) Compute $w_{2}^{k+1}$ according to (4.7).

3) Compute $z^{k+1}$ according to (4.8).

4) Compute $x^{k+1}$ by solving (4.9).

5) Compute $y^{k+1}$ by solving (4.10).

End Do

6) Update $\mu^{k+1}, v^{k+1}, \delta^{k+1}$, and $\kappa^{k+1}$ via (4.5).

This algorithm is just an application of the ADMM in nonsmooth settings with different scaling parameters. Mota et al. [27] established the global convergence of the method when the scaling parameters are equal to each other, but their proof can readily be extended to different scalings to produce the following theorem.

Theorem 4.1. Assume that $f \in \mathbb{R}^{n}$, the matrices $\nabla \in \mathbb{R}^{2 n \times n}, K \in \mathbb{R}^{n \times n}$ satisfy the condition (3.9), and $\mu_{L}$ and $\mu_{U}$ are defined by (3.10). If $\mu_{L}<\tau<\mu_{U}$, then the sequence $\left\{\left(x^{k}, y^{k}, z^{k}, w_{1}^{k}, w_{2}^{k} ; \mu^{k}, v^{k}, \delta^{k}, \kappa^{k}\right)\right\}$ generated by Algorithm 4.1 converges to $\left(\hat{x}, \hat{y}, \hat{z}, \hat{w}_{1}, \hat{w}_{2}\right.$; $\hat{\mu}, \hat{v}, \hat{\delta}, \hat{\kappa})$, where $\hat{x}$ is a solution of the problem (3.8). Moreover, $\hat{x}$ is also a solution of the problem (3.7) with $\lambda=\hat{\kappa}$. 
Remark 4.1. The equations in (4.2) can be written as

$$
\left(\begin{array}{cc}
\nabla & 0 \\
K & -I \\
K & I \\
0 & e^{\top}
\end{array}\right)\left(\begin{array}{l}
x \\
y
\end{array}\right)+\left(\begin{array}{ccc}
0 & 0 & -I \\
I & 0 & 0 \\
0 & -I & 0 \\
0 & 0 & 0
\end{array}\right)\left(\begin{array}{c}
w_{1} \\
w_{2} \\
z
\end{array}\right)=\left(\begin{array}{c}
0 \\
f \\
f \\
\tau
\end{array}\right) .
$$

Under the assumption (3.9), the coefficient matrices in (4.11) have full column rank, and all the corresponding constraints are polyhedral sets, hence the constrained model (4.2) is satisfied under the conditions of Theorem 1 in Ref. [27].

\section{Finding the Parameter $\tau$}

When using the constrained model (3.8) for the image recovery, one has to evaluate the parameter $\tau$, which can be done as follows:

- apply a filter $[16,26]$ to locate the set $\Omega^{C}$ with pixel values corrupted by impulsive noise;

- obtain the estimate $\hat{u}$ for $K \bar{x}$ by solving the model

$$
\begin{aligned}
& \min _{u}\|\nabla u\| \\
& \text { s.t. } u_{\Omega}=f_{\Omega} ;
\end{aligned}
$$

- compute the estimate for $\tau$ from the formula

$$
\tau:=\|\hat{u}-f\|_{1} .
$$

The main idea in this scheme is to use the solution of the model (5.1) as a replacement for $K \bar{x}$, in order to estimate $\tau$.

\section{Numerical Results}

Several constrained TV- $\ell_{1}$-problems were explored numerically, for two different datafidelity terms - viz. $\Phi_{\text {fit }}=\|K x-f\|_{1}$ and $\Phi_{\text {fit }}=\|S \cdot K x-f\|_{1}$. The code is written in MATLAB 7.12 (R2011a), and all the numerical experiments were conducted on a ThinkPad notebook with Intel Core i5-2140M CPU, a 2.3-GHz processor, and 4 GB of memory. The quality of the restoration was evaluated by considering the signal-to-noise ratio (SNR), measured in decibels $(\mathrm{dB})$ defined by

$$
\operatorname{SNR}(x) \triangleq 10 * \log _{10} \frac{\|\bar{x}-\tilde{x}\|^{2}}{\|\bar{x}-x\|^{2}},
$$

where $\bar{x}$ denotes the original image and $\tilde{x}$ the mean intensity value of $\bar{x}$. The recovered image quality was measured by the mean-square error (MSE). In all of the calculations, the algorithms were terminated if

$$
\text { Res }<5 \times 10^{-3} \text {. }
$$




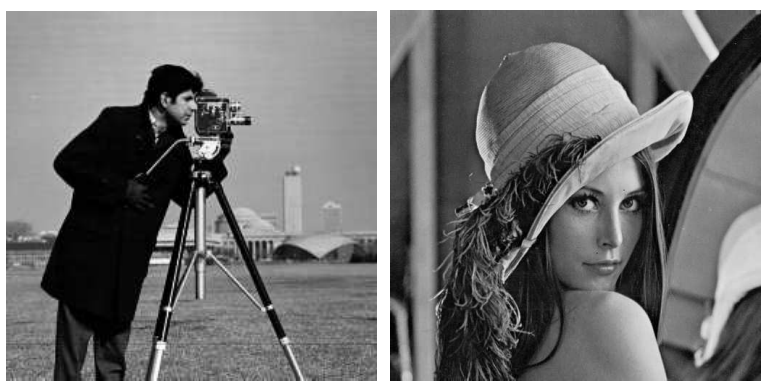

Figure 1: (a) Cameraman.tif, $256 \times 256$; (b) Lena.jpg, $512 \times 512$.

For the Algorithm 4.1, the residual Res is defined as

$$
\begin{aligned}
& \operatorname{Res}^{(1)}:=\max \left\{r_{x, z}, r_{x, y, w_{1}}, r_{x, y, w_{2}}, r_{y}\right\} \\
& r_{x, z}:=\nabla x-z \\
& r_{x, y, w_{1}}:=K x-f-y+w_{1}, \\
& r_{x, y, w_{2}}:=K x-f+y-w_{2}, \\
& r_{y}:=e^{\top} y-\tau .
\end{aligned}
$$

The FTVd solves the unconstrained TV- $\ell_{1}$-model $\min _{x} \sum_{i}\left\|\nabla_{i} x\right\|+\mu\|K x-f\|_{1}$ rewritten as

$$
\begin{array}{cl}
\min _{x, z_{i}, y} & \sum_{i}\left\|z_{i}\right\|+\mu\|y\|_{1} \\
\text { s.t. } & \nabla_{i} x=z_{i} \\
& K x-f=y
\end{array}
$$

by the ADMM, so the residual of the FTVd is defined by $\operatorname{Res}^{(2)}:=\max \left\{r_{x, z}, r_{x, y}\right\}$ and

$$
r_{x, z}:=\nabla x-z, \quad r_{x, y}:=K x-f-y .
$$

In the simulations, both salt-and-pepper and random-valued impulse noise were considered, in seeking to establish the following:

- the scheme of Section 5 produces good estimates of $\tau$ in constrained models;

- Theorem 3.1(ii) is valid for TV- $\ell_{1}$-models - i.e. the quality of the images restored by unconstrained models, with $\lambda$ determined in Theorem 4.1, is comparable with constrained models; and

- the regularisation parameter is on par with one determined by the trial-and-error method.

The tested images are shown in Fig. 1. 


\subsection{Deblurring from impulsive noise}

The first focus was on models with the data-fidelity term $\Phi_{\text {fit }}=\|K x-f\|_{1}$. Three sets of tests were used to verify the above three considerations to: (1) show the efficiency of the method (5.1)-(5.2) in the selection of a suitable parameter $\tau ; 2)$ compare the SNR of the images restored by the constrained and unconstrained models and (3) compare the "optimal" regularisation parameter of the unconstrained model determined by the trialand-error method and Algorithm 4.1.

The vector $f$ was generated in three steps: (1) all pixel values were scaled to the interval $[0,1]$; (2) the test images were blurred with the blurring (I)-fspecial ('average', 9) and (II)-fspecial ('gaussian', [9 9],3); and (3) the resulting images were corrupted by impulse noise at different levels. All algorithms used such blurred images initially. The first set of tests dealt with blurred and noisy images degraded under periodic boundary conditions, with the impulse noise at the level $30 \%, 40 \%, 50 \%$ or $60 \%$ added. For the saltand-pepper noise, the noise candidate set $\Omega^{C}$ was detected by the AMF algorithm [26]. The maximum window size in the AMF algorithm was set to 19, as suggested in Ref. [26]. For the random-value impulsive noise, the set $\Omega^{C}$ was identified by the statistical method with a default setting [16]. The model (5.1) with the obtained $\Omega$ was then solved by applying the ADMM, reformulated as follows:

$$
\begin{array}{ll}
\min _{u, z, y}\|y\| & \\
\text { s.t. } & \nabla u=y \\
& u=z \\
& z_{\Omega}=f_{\Omega} .
\end{array}
$$

The penalty parameters $\beta_{1}$ and $\beta_{2}$ in the ADMM method related to the first and second equations in the model (6.2) were set to 10 and 20, respectively. The solution $\hat{u}$ of (6.2) was used to calculate $\tau_{2}$ by the formula (5.2). Table 1 shows $\tau_{1}:=\|K \bar{x}-f\|_{1}, \tau_{2}$ and the relative error $\left|\tau_{1}-\tau_{2}\right| / \tau_{1}$ for each case considered. The computed value $\tau_{2}$ is comparable with the true value $\tau_{1}$ used in the constrained models.

The Algorithm 4.1 and the FTVd of Ref. [40] were used to solve the constrained and unconstrained TV- $\ell_{1}$-models, respectively. In the second set of tests, blurred and noisy images were degraded under periodic boundary conditions, and impulse noise at the level $30 \%, 40 \%, 50 \%$ and $60 \%$ was added. The parameters $\beta_{1}=200, \beta_{2}=10$, and $\beta_{3}=200$ in Algorithm 4.1 produce satisfactory results for all tests. In the FTVd, the same penalty parameters were chosen as in Algorithm 4.1 - i.e. the respective penalty parameters for the equations $\nabla x-z=0$ and $K x-f-y=0$ were $\beta_{1}=200$ and $\beta_{3}=200$. Under Algorithm 4.1, the restored image and the last iteration for $\hat{\kappa}$ were obtained simultaneously, and then the $\mathrm{FTVd}^{\dagger}$ with the derived parameter $\hat{\kappa}$ and the stopping criterion (6.1), to find the solution of the TV- $\ell_{1}$-model (3.7). The values of the parameter $\hat{\kappa}$ and SNRs for the constrained and unconstrained models are presented in Table 2. The recovered MSEs are

${ }^{\dagger}$ http://www.caam.rice.edu/ optimization/L1/ftvd/v4.0/ 
Table 1: The values of $\tau_{1}$ and $\tau_{2}$.

\begin{tabular}{|c|c|c|c|c|c|}
\hline Tested image & Blur & noise level & $\tau_{1}:=\|K \bar{x}-f\|_{1}$ & $\tau_{2}$ via $(5.1)-(5.2)$ & $\left|\tau_{1}-\tau_{2}\right| / \tau_{1}$ \\
\hline \multicolumn{6}{|c|}{ salt-and-pepper impulse noise } \\
\hline \multirow[t]{8}{*}{$\mathrm{a}$} & \multirow[t]{4}{*}{ I } & $30 \%$ & $9.8196 \mathrm{e}+003$ & $9.8196 \mathrm{e}+003$ & $2.3464 \mathrm{e}-006$ \\
\hline & & $40 \%$ & $1.3087 \mathrm{e}+004$ & $1.3086 \mathrm{e}+004$ & $7.3590 \mathrm{e}-005$ \\
\hline & & $50 \%$ & $1.6301 \mathrm{e}+004$ & $1.6302 \mathrm{e}+004$ & $9.1184 \mathrm{e}-005$ \\
\hline & & $60 \%$ & $1.9643 e+004$ & $1.9642 \mathrm{e}+004$ & $4.9575 e-005$ \\
\hline & \multirow[t]{4}{*}{ II } & $30 \%$ & $9.8209 e+003$ & $9.8206 e+003$ & $2.5360 \mathrm{e}-005$ \\
\hline & & $40 \%$ & $1.3097 \mathrm{e}+004$ & $1.3096 \mathrm{e}+004$ & $3.9853 e-005$ \\
\hline & & $50 \%$ & $1.6455 e+004$ & $1.6454 \mathrm{e}+004$ & $3.9977 \mathrm{e}-005$ \\
\hline & & $60 \%$ & $1.9515 e+004$ & $1.9515 e+004$ & $5.1470 \mathrm{e}-006$ \\
\hline \multicolumn{6}{|c|}{ random value impulse noise } \\
\hline \multirow[t]{8}{*}{$\mathrm{a}$} & \multirow[t]{4}{*}{ I } & $30 \%$ & $9.9073 e+003$ & $9.9063 e+003$ & $1.0360 \mathrm{e}-004$ \\
\hline & & $40 \%$ & $1.3155 e+004$ & $1.3156 \mathrm{e}+004$ & $9.6410 \mathrm{e}-005$ \\
\hline & & $50 \%$ & $1.6382 \mathrm{e}+004$ & $1.6383 e+004$ & $7.2474 \mathrm{e}-005$ \\
\hline & & $60 \%$ & $1.9691 \mathrm{e}+004$ & $1.9687 \mathrm{e}+004$ & $1.9382 \mathrm{e}-004$ \\
\hline & \multirow[t]{4}{*}{ II } & $30 \%$ & $1.3190 \mathrm{e}+004$ & $1.3192 \mathrm{e}+004$ & $1.5832 \mathrm{e}-004$ \\
\hline & & $40 \%$ & $1.3090 \mathrm{e}+004$ & $1.3089 \mathrm{e}+004$ & $7.3576 \mathrm{e}-005$ \\
\hline & & $50 \%$ & $1.6403 e+004$ & $1.6404 \mathrm{e}+004$ & 2.9497e-005 \\
\hline & & $60 \%$ & $1.9590 \mathrm{e}+004$ & $1.9593 e+004$ & $1.6469 \mathrm{e}-004$ \\
\hline \multicolumn{6}{|c|}{ salt-and-pepper impulse noise } \\
\hline \multirow[t]{8}{*}{$\mathrm{b}$} & \multirow[t]{4}{*}{$\mathrm{I}$} & $30 \%$ & $9.8691 \mathrm{e}+003$ & $9.8692 \mathrm{e}+003$ & $1.0326 \mathrm{e}-005$ \\
\hline & & $40 \%$ & $1.3183 e+004$ & $1.3184 \mathrm{e}+004$ & $3.0565 \mathrm{e}-005$ \\
\hline & & $50 \%$ & $1.6373 e+004$ & $1.6373 e+004$ & $2.4667 e-005$ \\
\hline & & $60 \%$ & $1.9641 \mathrm{e}+004$ & $1.9642 \mathrm{e}+004$ & $1.2129 \mathrm{e}-005$ \\
\hline & \multirow[t]{4}{*}{ II } & $30 \%$ & $9.8681 e+003$ & $9.8691 \mathrm{e}+003$ & $1.0684 \mathrm{e}-004$ \\
\hline & & $40 \%$ & $1.3157 \mathrm{e}+004$ & $1.3157 \mathrm{e}+004$ & $1.9127 \mathrm{e}-005$ \\
\hline & & $50 \%$ & $1.6319 e+004$ & $1.6319 e+004$ & $2.2055 \mathrm{e}-006$ \\
\hline & & $60 \%$ & $1.9668 \mathrm{e}+004$ & $1.9668 \mathrm{e}+004$ & $1.1229 \mathrm{e}-005$ \\
\hline \multicolumn{6}{|c|}{ random value impulse noise } \\
\hline \multirow[t]{8}{*}{$\mathrm{b}$} & \multirow[t]{4}{*}{$\mathrm{I}$} & $30 \%$ & $9.7770 \mathrm{e}+003$ & $9.7770 \mathrm{e}+003$ & $7.8120 \mathrm{e}-007$ \\
\hline & & $40 \%$ & $1.3067 \mathrm{e}+004$ & $1.3066 \mathrm{e}+004$ & $9.2740 \mathrm{e}-005$ \\
\hline & & $50 \%$ & $1.6360 \mathrm{e}+004$ & $1.6361 \mathrm{e}+004$ & $5.5121 \mathrm{e}-005$ \\
\hline & & $60 \%$ & $1.9709 \mathrm{e}+004$ & $1.9709 \mathrm{e}+004$ & $1.9229 \mathrm{e}-005$ \\
\hline & \multirow[t]{4}{*}{ II } & $30 \%$ & $9.8392 \mathrm{e}+003$ & $9.8397 e+003$ & $5.1033 e-005$ \\
\hline & & $40 \%$ & $1.3113 e+004$ & $1.3114 \mathrm{e}+004$ & $2.2270 \mathrm{e}-005$ \\
\hline & & $50 \%$ & $1.6347 \mathrm{e}+004$ & $1.6348 \mathrm{e}+004$ & $6.2731 \mathrm{e}-005$ \\
\hline & & $60 \%$ & $1.9659 \mathrm{e}+004$ & $1.9661 \mathrm{e}+004$ & $8.3863 e-005$ \\
\hline
\end{tabular}

the same for constrained and unconstrained models, so not reported here. Fig. 2 shows the images degraded by the fspecial ('average', 9) blur along with the salt-and-pepper impulse noise with the images restored via the two models. The results are much the same as found in other cases.

In the third set of tests, the degraded images were generated similarly. By sampling a large range of the regularisation parameter $\lambda$ that also contained $\hat{\kappa}$, the FTVd was run with different regularisation parameters to identify the particular "optimal" value $\lambda^{\text {opt }}$ that 
Table 2: Numerical results on the models (3.7) and (3.8).

\begin{tabular}{|c|c|c|c|c|c|c|c|}
\hline $\begin{array}{c}\text { Tested } \\
\text { image }\end{array}$ & Blur & $\begin{array}{l}\text { noise } \\
\text { level }\end{array}$ & $\begin{array}{l}\text { SNR }(d B) \\
\text { via }(3.8)\end{array}$ & $\hat{\kappa}$ & $\begin{array}{l}\text { SNR (dB) via } \\
\text { (3.7) with } \hat{\kappa}\end{array}$ & $\lambda^{\text {opt }}$ & $\begin{array}{l}\text { SNR }(\mathrm{dB}) \text { via } \\
\text { (3.7) with } \lambda^{\text {opt }}\end{array}$ \\
\hline \multicolumn{8}{|c|}{ salt-and-pepper impulse noise } \\
\hline \multirow[t]{8}{*}{$\mathrm{a}$} & \multirow[t]{4}{*}{ I } & $30 \%$ & 17.519 & 59.090 & 17.401 & 70 & 17.4636 \\
\hline & & $40 \%$ & 14.720 & 22.473 & 14.658 & 46 & 15.5418 \\
\hline & & $50 \%$ & 13.631 & 21.484 & 13.598 & 32 & 13.8932 \\
\hline & & $60 \%$ & 12.404 & 18.623 & 12.375 & 21 & 12.3928 \\
\hline & \multirow[t]{4}{*}{ II } & $30 \%$ & 16.299 & 63.131 & 16.077 & 108 & 16.4733 \\
\hline & & $40 \%$ & 13.741 & 22.013 & 13.678 & 69 & 14.7644 \\
\hline & & $50 \%$ & 13.155 & 21.667 & 13.122 & 49 & 13.7138 \\
\hline & & $60 \%$ & 12.381 & 19.881 & 12.359 & 32 & 12.5819 \\
\hline \multicolumn{8}{|c|}{ random value impulse noise } \\
\hline \multirow[t]{8}{*}{$\mathrm{a}$} & \multirow[t]{4}{*}{$\mathrm{I}$} & $30 \%$ & 17.037 & 51.374 & 16.893 & 60 & 17.003 \\
\hline & & $40 \%$ & 13.344 & 20.706 & 13.020 & 19 & 13.198 \\
\hline & & $50 \%$ & 10.729 & 12.856 & 10.663 & 9 & 10.898 \\
\hline & & $60 \%$ & 7.426 & 2.503 & 7.499 & 5 & 7.795 \\
\hline & \multirow[t]{4}{*}{ II } & $30 \%$ & 15.784 & 56.051 & 15.716 & 89 & 16.214 \\
\hline & & $40 \%$ & 12.356 & 19.221 & 12.229 & 33 & 12.375 \\
\hline & & $50 \%$ & 10.762 & 8.797 & 10.776 & 6 & 10.869 \\
\hline & & $60 \%$ & 7.406 & 2.280 & 7.549 & 5 & 7.872 \\
\hline \multicolumn{8}{|c|}{ salt-and-pepper impulse noise } \\
\hline \multirow[t]{8}{*}{$\mathrm{b}$} & \multirow[t]{4}{*}{ I } & $30 \%$ & 16.861 & 55.512 & 16.820 & 60 & 16.828 \\
\hline & & $40 \%$ & 15.070 & 18.258 & 14.983 & 35 & 15.4508 \\
\hline & & $50 \%$ & 14.239 & 17.263 & 14.189 & 25 & 14.3542 \\
\hline & & $60 \%$ & 13.186 & 14.919 & 13.136 & 18 & 13.1740 \\
\hline & \multirow[t]{4}{*}{ II } & $30 \%$ & 16.254 & 57.615 & 15.958 & 82 & 16.0503 \\
\hline & & $40 \%$ & 14.521 & 17.585 & 14.428 & 42 & 14.8931 \\
\hline & & $50 \%$ & 14.040 & 17.548 & 13.963 & 34 & 14.2696 \\
\hline & & $60 \%$ & 13.249 & 16.573 & 13.179 & 25 & 13.348 \\
\hline \multicolumn{8}{|c|}{ random value impulse noise } \\
\hline \multirow[t]{8}{*}{$\mathrm{b}$} & \multirow[t]{8}{*}{ I } & $30 \%$ & 16.712 & 49.937 & 16.471 & 58 & 16.498 \\
\hline & & $40 \%$ & 14.313 & 17.692 & 14.246 & 24 & 14.385 \\
\hline & & $50 \%$ & 12.068 & 14.117 & 12.029 & 13 & 12.035 \\
\hline & & $60 \%$ & 8.607 & 4.665 & 8.575 & 6 & 8.693 \\
\hline & & $30 \%$ & 15.954 & 52.114 & 15.770 & 73 & 15.868 \\
\hline & & $40 \%$ & 13.947 & 17.147 & 13.887 & 25 & 14.060 \\
\hline & & $50 \%$ & 11.657 & 13.026 & 11.603 & 11 & 11.772 \\
\hline & & $60 \%$ & 8.580 & 3.676 & 8.620 & 4 & 8.696 \\
\hline
\end{tabular}

provided the highest SNR of the restored image. The results are also displayed in Table 2, where the optimal value $\lambda^{o p t}$ and SNR of the recovered image are listed. For the saltand-pepper impulse noise, the discrepancy between the SNRs of the images recovered via $\hat{\kappa}$ and $\lambda^{o p t}$ is small. On the other hand, for the random-valued impulsive noise there is a clear difference between the SNRs for images recovered by constrained and "optimal" unconstrained models. 

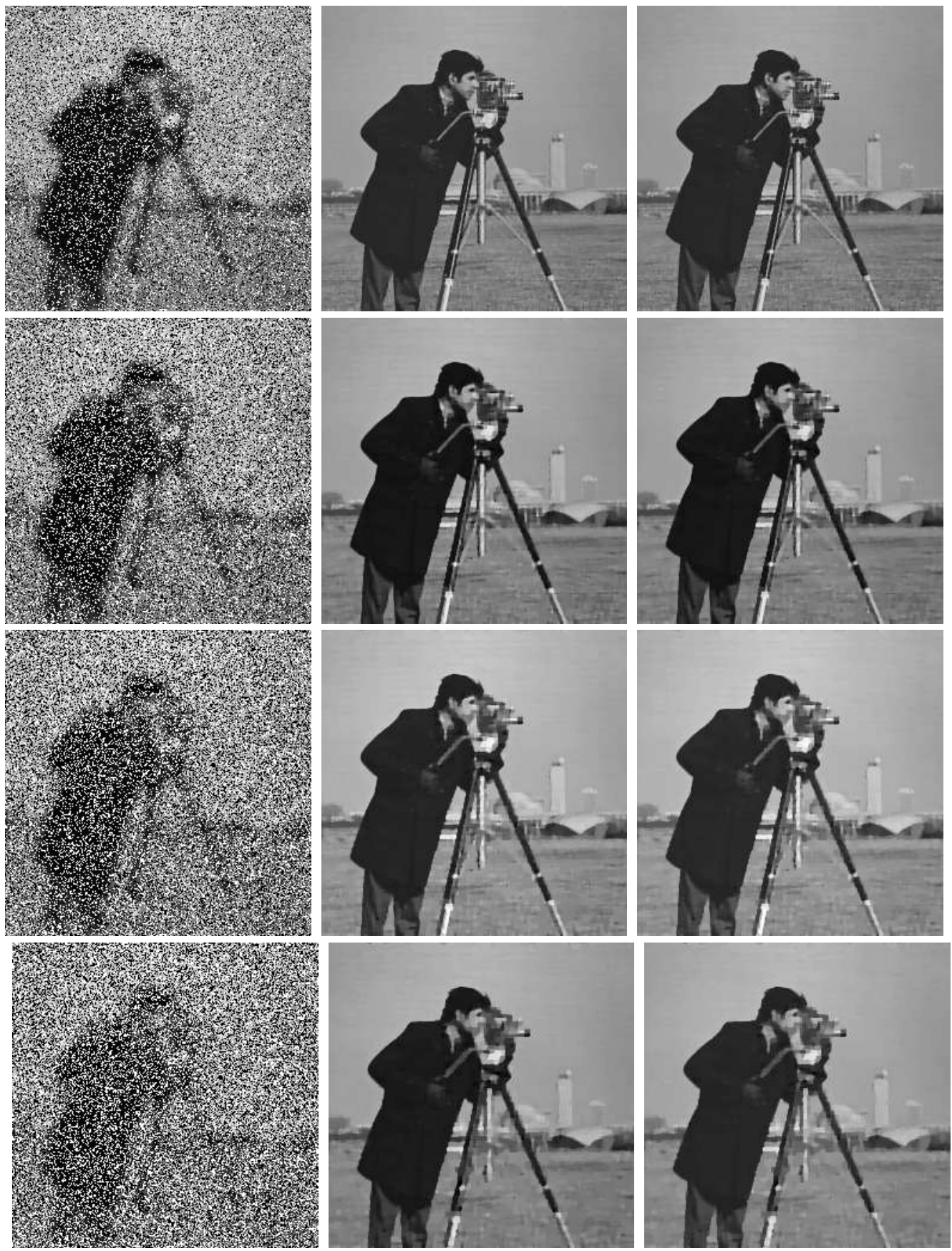

Figure 2: Left: Blurred and noisy image; Middle: Images restored via constrained model (3.8); Right: Images restored via unconstrained model (3.7). Noise level: Row 1 - 30\%; Row 2 - 40\%; Row 3 - 50\%; Row $4-60 \%$. 

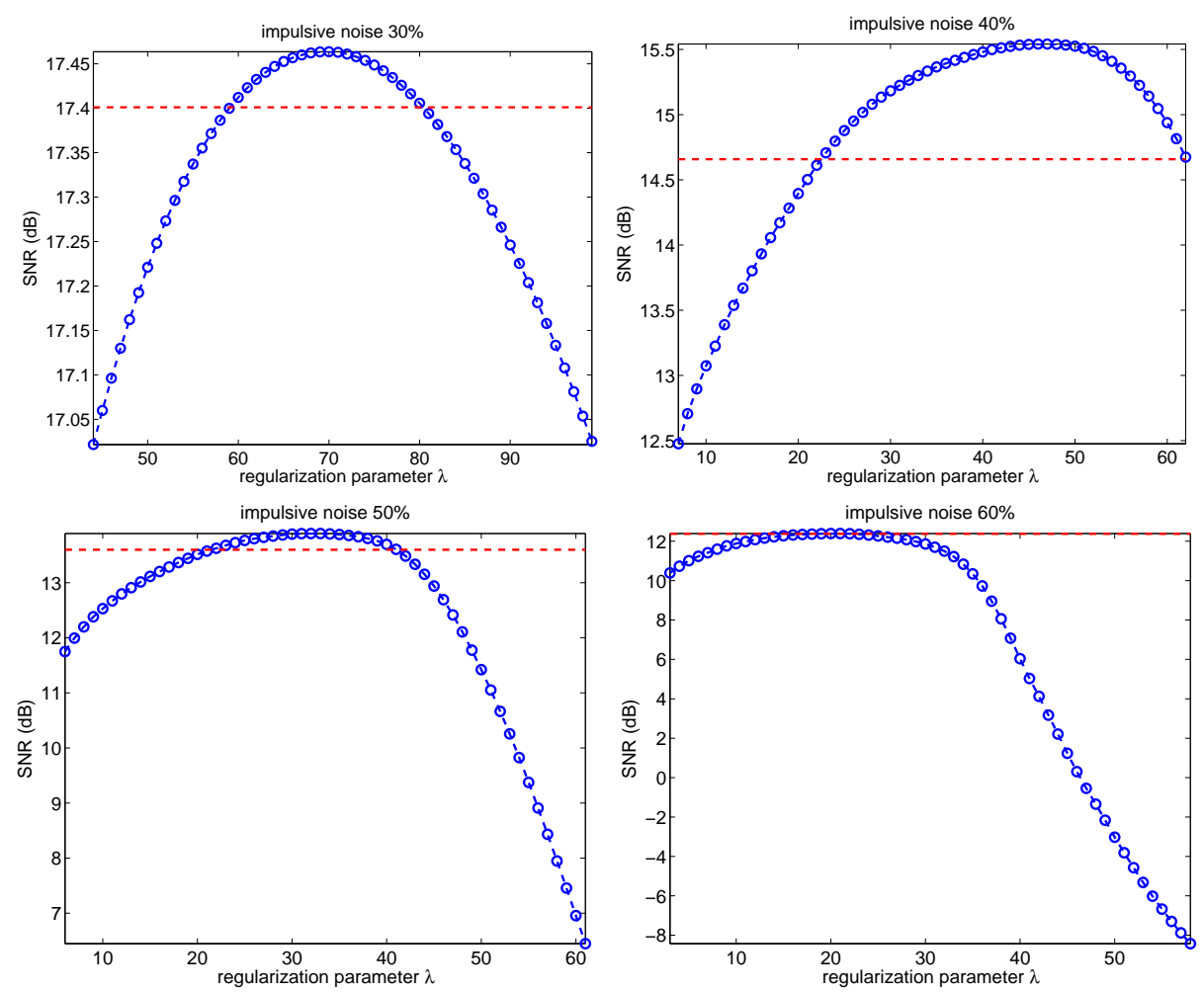

Figure 3: Restored SNR against $\lambda$, and the difference between the restored via $\hat{\kappa}$ and the trial-and-error method; Noise level: Row $1-30 \%$ and 40\%; Row $2-50 \%$ and $60 \%$. Noise: salt-and-pepper; Blur - I.

In Fig. 3, graphs of SNRs for the unconstrained TV- $\ell_{1}$-model (3.7) with different regularisation parameters are shown. The red dashed line represents the SNR with $\hat{\kappa}$ as the regularisation parameter. The curve over the red dashed line shows the gap between the SNRs for images recovered via $\hat{\kappa}$ and the "optimal" value found. The graphs in Fig. 3 correspond to a different level of salt-and-pepper noise. For the "Cameraman.tif" image degraded by fspecial ('average', 9) blur, for low and middle noise levels the discrepancy between the SNRs for $\hat{\kappa}$ and "optimal" values is about $0.2 \mathrm{~dB}$, and diminishes for higher noise levels. The results of numerical experiments for the Neumann boundary conditions were similar, so not reported here.

\subsection{Inpainting from impulsive noise}

The efficiency of the approach was explored for the models (1.2) and (1.3) with the data-fidelity term $\Phi_{\text {fit }}=\|S K x-f\|_{1}$. The resulting models

$$
\min _{x}\|\nabla x\|+\lambda\|S K x-f\|_{1}
$$




$$
\begin{aligned}
& \min _{x}\|\nabla x\| \\
& \text { s.t. }\|S K x-f\|_{1} \leq \tau,
\end{aligned}
$$

are suitable for image inpainting from impulsive noise. The parameter $\tau$ in the model (6.4) can be determined analogously to Section 5 as follows:

- apply a filter from Ref. [16] to locate the set $\Pi^{C}$ where the pixel values are corrupted by impulsive noise;

- obtain the estimate $\hat{u}$ of $K \bar{x}$ by solving the model

$$
\begin{aligned}
& \min _{u}\|\nabla u\| \\
& \text { s.t. } \quad(S u)_{\Pi}=f_{\Pi} ;
\end{aligned}
$$

- compute the estimate for $\tau$ from the formula

$$
\tau:=\|(S \hat{u})-f\|_{1} .
$$

In the first set of tests, $\tau$ was obtained from the model (6.5) and Eq. (6.6). The images were degraded by blur and noise under periodic boundary conditions, and different sample ratios ranging from $30 \%$ to $70 \%$ with equal $10 \%$ distance were tested, followed by salt-andpepper impulsive noise. The noise level was set to 5\% and 15\%, and the model (6.5) solved by the ADMM reformulated as

$$
\begin{array}{ll}
\min _{u, y, w, z}\|y\| \\
\text { s.t. } & \nabla u=y \\
& u=w \\
& S u=z \\
& z_{\Pi}=f_{\Pi},
\end{array}
$$

where the chosen penalty parameter values in the ADMM were $\beta_{1}=10, \beta_{2}=20$, and $\beta_{3}=20$. Recall that $\beta_{1}, \beta_{2}$, and $\beta_{3}$ are related to the first, second, and third equations in (6.7), respectively. The solution $\hat{u}$ of (6.7) was obtained, and then computed $\tau_{2}$ using the formula (6.6). The results are presented in Table 3, where the parameter $\tau_{2}$ is seen to be a good approximation for the true value $\tau_{1}$, so it was used in subsequent tests for the constrained model (6.4). The details of these tests are discussed in the Appendix, where the procedure from Section 6.1 was followed. Thus two types of impulsive noise and two types of the blur were considered, and vector $f$ generated as follows. A convolution $K$ was applied to $\bar{x}$ to obtain $K \bar{x}$ under the periodic boundary condition, and random samples were taken to get $S K \bar{x}$. Various sample ratios ranging from $30 \%$ to $70 \%$ with the equal distance $10 \%$ were tested, and then the impulsive noise added with the noise level set to $5 \%$ and $15 \%$. The SNRs of the images restored by constrained and unconstrained models were compared, and the "optimal" regularisation parameter obtained was used in the corresponding unconstrained model. 
Table 3: The values of $\tau_{1}$ and $\tau_{2}$.

\begin{tabular}{|c|c|c|c|c|c|}
\hline Tested image & Blur & sample ratio & $\tau_{1}:=\|S K \bar{x}-f\|_{1}$ & $\tau_{2}$ via (6.5)-(6.6) & $\left|\tau_{1}-\tau_{2}\right| / \tau_{1}$ \\
\hline \multicolumn{5}{|c|}{ salt-and-pepper (noise level - 5\%) } \\
\hline a & I & $30 \%$ & $8.4583 \mathrm{e}+002$ & $8.4670 \mathrm{e}+002$ & $1.0285 \mathrm{e}-003$ \\
& & $40 \%$ & $1.1467 \mathrm{e}+003$ & $1.1469 \mathrm{e}+003$ & $1.8674 \mathrm{e}-004$ \\
& & $50 \%$ & $1.3933 \mathrm{e}+003$ & $1.3936 \mathrm{e}+003$ & $2.4051 \mathrm{e}-004$ \\
& & $60 \%$ & $1.6902 \mathrm{e}+003$ & $1.6908 \mathrm{e}+003$ & $3.6453 \mathrm{e}-004$ \\
& & $70 \%$ & $1.9872 \mathrm{e}+003$ & $1.9879 \mathrm{e}+003$ & $3.0933 \mathrm{e}-004$ \\
& II & $30 \%$ & $8.6568 \mathrm{e}+002$ & $8.6533 \mathrm{e}+002$ & $4.1154 \mathrm{e}-004$ \\
& & $40 \%$ & $1.1611 \mathrm{e}+003$ & $1.1615 \mathrm{e}+003$ & $3.3930 \mathrm{e}-004$ \\
& & $50 \%$ & $1.4558 \mathrm{e}+003$ & $1.4558 \mathrm{e}+003$ & $3.1312 \mathrm{e}-005$ \\
& & $60 \%$ & $1.7513 \mathrm{e}+003$ & $1.7515 \mathrm{e}+003$ & $1.0286 \mathrm{e}-004$ \\
& & $70 \%$ & $2.0249 \mathrm{e}+003$ & $2.0250 \mathrm{e}+003$ & $7.6999 \mathrm{e}-005$ \\
\hline & I & $30 \%$ & $2.5571 \mathrm{e}+003$ & $2.5572 \mathrm{e}+003$ & $4.4790 \mathrm{e}-005$ \\
& & $40 \%$ & $3.4058 \mathrm{e}+003$ & $3.4076 \mathrm{e}+003$ & $5.1017 \mathrm{e}-004$ \\
& & $50 \%$ & $4.2525 \mathrm{e}+003$ & $4.2529 \mathrm{e}+003$ & $9.7112 \mathrm{e}-005$ \\
& & $60 \%$ & $5.1351 \mathrm{e}+003$ & $5.1353 \mathrm{e}+003$ & $3.6894 \mathrm{e}-005$ \\
& & $70 \%$ & $5.9105 \mathrm{e}+003$ & $5.9107 \mathrm{e}+003$ & $3.5500 \mathrm{e}-005$ \\
& II & $30 \%$ & $2.5622 \mathrm{e}+003$ & $2.5630 \mathrm{e}+003$ & $3.1496 \mathrm{e}-004$ \\
& & $40 \%$ & $3.4350 \mathrm{e}+003$ & $3.4347 \mathrm{e}+003$ & $6.6886 \mathrm{e}-005$ \\
& & $50 \%$ & $4.3173 \mathrm{e}+003$ & $4.3171 \mathrm{e}+003$ & $5.2739 \mathrm{e}-005$ \\
& & $60 \%$ & $5.1593 \mathrm{e}+003$ & $5.1601 \mathrm{e}+003$ & $1.6458 \mathrm{e}-004$ \\
& & $70 \%$ & $6.0577 \mathrm{e}+003$ & $6.0574 \mathrm{e}+003$ & $4.3713 \mathrm{e}-005$ \\
\hline
\end{tabular}

The ADMM from Ref. [38] was used to solve the unconstrained model (6.3), and the results are presented in Table 4. It is notable that SNRs for the images recovered by these models are almost the same. Fig. 4 shows the restoration results for the constrained model and the penalised version for the sample ratios $30 \%, 40 \%, 50 \%$ and $60 \%$.

Fig. 5 shows graphs of the SNR for the image restored by the penalised version (6.3) against $\lambda$. The red dashed lines represent the SNRs for $\lambda=\hat{\kappa}$ in the penalised model (6.3), and the curves over the red dashed lines indicate the gap between the SNRs of the images recovered via the parameter $\hat{\kappa}$ and via the optimal recovered value. It is again notable that the gap diminishes as the sample ratio increases.

\section{Conclusions}

Minimisation of the seminorms $\|D \cdot\|$ for $\ell_{1}$-data-fidelity terms has been considered, where the solution of the discrepancy equation is not unique. Connexions between constrained and unconstrained models have been established, and the optimal regularisation parameter evaluated by solving constrained model. This approach preserves a closed-form solution of each subproblem, and recovers blurry images with simultaneous evaluation of the balance parameter of the unconstrained model. The numerical simulations illustrate the efficiency of the method for constrained $\ell_{1}$-models. 

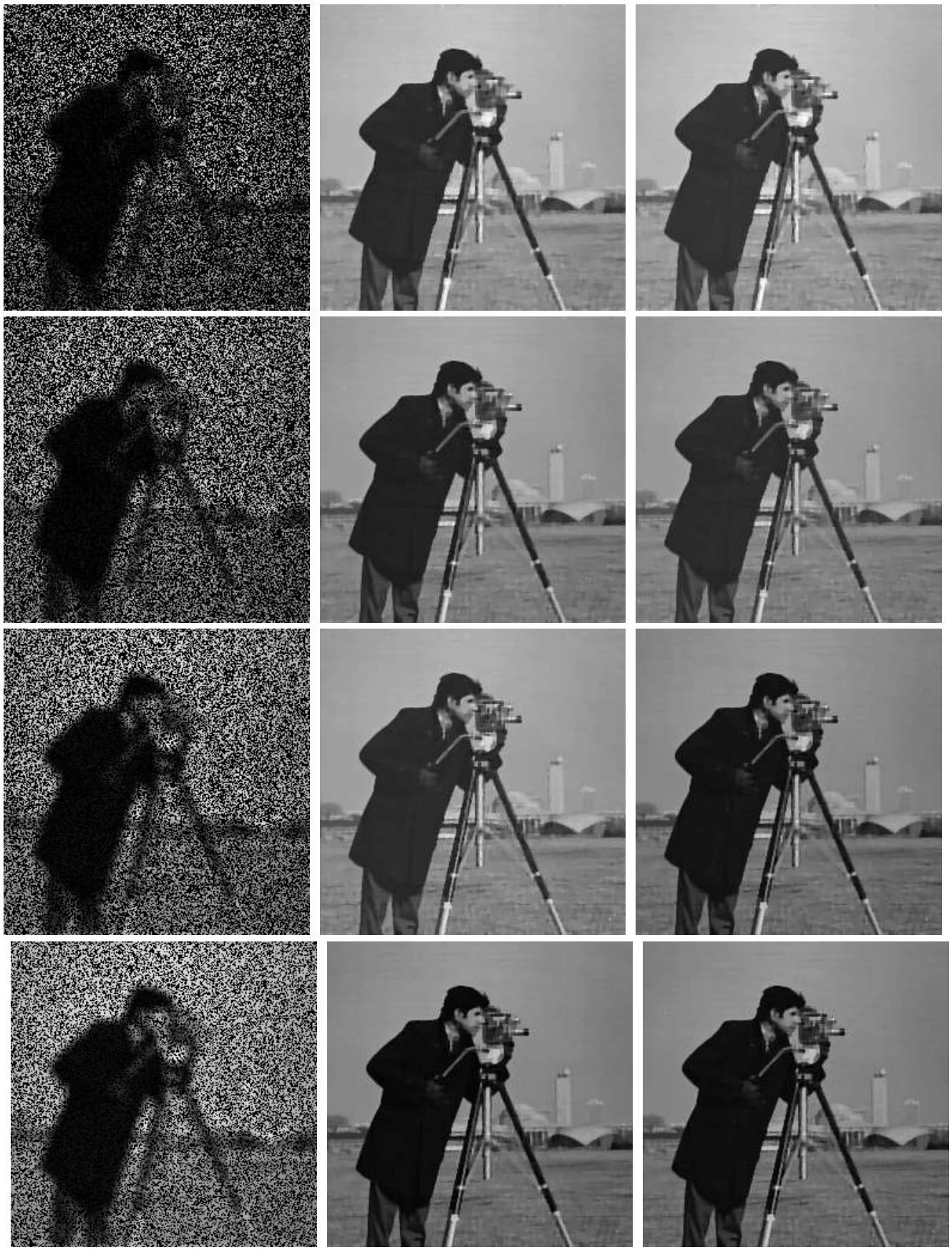

Figure 4: Left: Blurred and noisy images. Middle: Images restored by the model (6.4). Right: Images restored by the model (6.3). Sample ratio: Row $1-30 \%$; Row 2-40\%; Row 3-50\%; Row $4-60 \%$. Noise level $-5 \%$. Blur -1 . 
Table 4: Numerical results for the models (6.3) and (6.4).

\begin{tabular}{|c|c|c|c|c|c|c|c|}
\hline $\begin{array}{l}\text { Tested } \\
\text { image }\end{array}$ & Blur & $\begin{array}{l}\text { noise } \\
\text { level }\end{array}$ & $\begin{array}{l}\text { SNR (dB) } \\
\text { via }(6.4)\end{array}$ & $\hat{\kappa}$ & $\begin{array}{l}\text { SNR (dB) via } \\
\text { (6.3) with } \hat{\kappa}\end{array}$ & $\lambda^{\text {opt }}$ & $\begin{array}{l}\text { SNR (dB) via } \\
\text { (6.3) with } \lambda^{o p t}\end{array}$ \\
\hline \multicolumn{8}{|c|}{ salt-and-pepper (noise level: 5\%) } \\
\hline \multirow[t]{10}{*}{$\mathrm{a}$} & \multirow[t]{5}{*}{$\overline{\mathrm{I}}$} & $30 \%$ & 14.063 & 38.582636 & 14.028 & 67 & 14.192 \\
\hline & & $40 \%$ & 14.885 & 34.529045 & 14.808 & 66 & 15.070 \\
\hline & & $50 \%$ & 15.448 & 31.441490 & 15.358 & 66 & 15.528 \\
\hline & & $60 \%$ & 15.806 & 28.988864 & 15.724 & 63 & 15.874 \\
\hline & & $70 \%$ & 16.227 & 27.282183 & 16.150 & 61 & 16.291 \\
\hline & \multirow[t]{5}{*}{ II } & $30 \%$ & 13.163 & 32.655956 & 13.116 & 67 & 13.277 \\
\hline & & $40 \%$ & 13.635 & 30.981656 & 13.595 & 65 & 13.759 \\
\hline & & $50 \%$ & 13.986 & 29.048234 & 13.932 & 64 & 14.132 \\
\hline & & $60 \%$ & 14.278 & 27.365302 & 14.213 & 62 & 14.515 \\
\hline & & $70 \%$ & 14.569 & 26.609349 & 14.507 & 61 & 14.731 \\
\hline \multicolumn{8}{|c|}{ salt-and-pepper (noise level: 15\%) } \\
\hline \multirow[t]{10}{*}{$\mathrm{a}$} & \multirow[t]{5}{*}{$\overline{\mathrm{I}}$} & $30 \%$ & 13.423 & 34.766208 & 13.377 & 60 & 13.595 \\
\hline & & $40 \%$ & 14.125 & 31.727550 & 14.065 & 55 & 14.376 \\
\hline & & $50 \%$ & 14.611 & 27.201878 & 14.529 & 55 & 14.761 \\
\hline & & $60 \%$ & 15.060 & 27.201878 & 14.986 & 47 & 15.229 \\
\hline & & $70 \%$ & 15.659 & 25.884016 & 15.554 & 47 & 15.790 \\
\hline & \multirow[t]{5}{*}{ II } & $30 \%$ & 12.700 & 27.544485 & 12.671 & 62 & 12.938 \\
\hline & & $40 \%$ & 13.126 & 27.701021 & 13.095 & 60 & 13.381 \\
\hline & & $50 \%$ & 13.595 & 26.514622 & 13.547 & 61 & 13.816 \\
\hline & & $60 \%$ & 13.802 & 25.701829 & 13.748 & 60 & 14.016 \\
\hline & & $70 \%$ & 14.136 & 24.938766 & 14.063 & 55 & 14.353 \\
\hline
\end{tabular}

\section{Acknowledgments}

The author was supported by the Natural Science Foundation of China (Grant No. NSFC-1130128) and the sponsorship of Jiangsu overseas research and training program for university prominent young and middle-aged teachers and presidents. The author would like to thank the referees and the associate editor to improve the manuscript.

\section{Appendix}

Some basic information about image inpainting is provided here, involving the infill of missing or damaged regions in an image - cf. also Ref. [12]. Let $\bar{x}$ be an unknown image. The observed image $f$ after image inpainting is given by

$$
f=N(S(K \bar{x})),
$$

where $S \in \mathbb{R}^{n \times n}$ is a mask operator and the corresponding constrained $\ell_{1}$-model (6.4) is similar to that in Refs. $[11,13]$. If $S K$ is considered the whole matrix $K^{\prime}$, under Algorithm 4.1 the $x$-related subproblem does not have a closed-form solution as the matrices on the left-hand side of equation (4.9) are not diagonalisable by a discrete FFT or discrete DCT. 

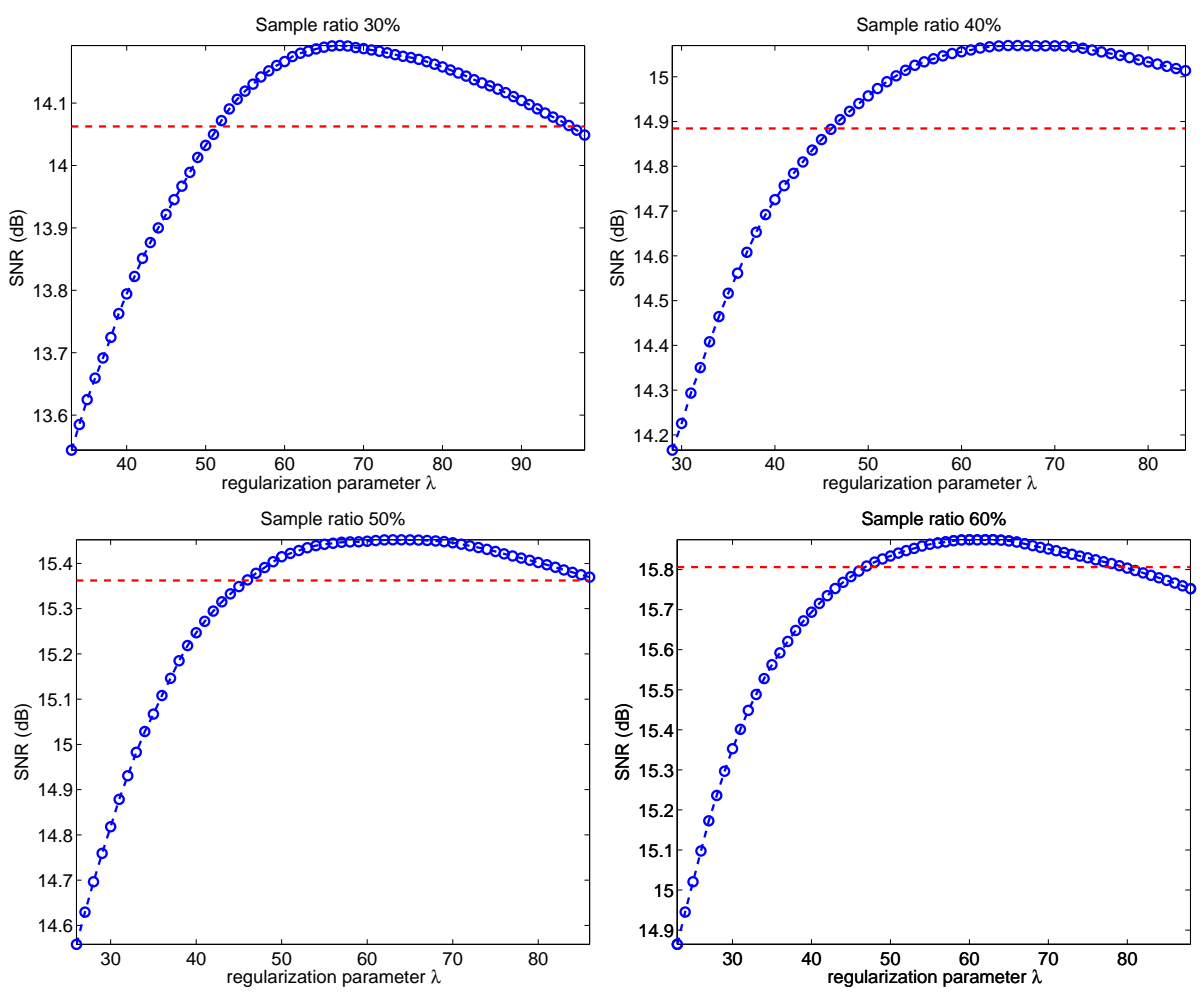

Figure 5: Restored SNR vs. $\lambda$, and difference between the restored SNR with $\hat{\kappa}$ and trial-and-error. Sample ratio: Row $1-30 \%$ and 40\%; Row $2-50 \%$ and $60 \%$. Noise level: $5 \%$. Blur - I.

However, one can rewrite the model (6.4) as

$$
\begin{array}{lll}
\min _{x, y, z_{i}, v, u_{1}, u_{2}, w} \sum_{i=1}^{n}\left\|z_{i}\right\| & \\
\text { s.t. } & \nabla_{i} x=z_{i} & : \mu_{i} \\
& K x=v & : p_{1} \\
& S v-f=y & : p_{2} \\
y+u_{1}-w=0 & : v \\
y-u_{2}+w=0 & : \delta \\
& e^{\top} w=\tau & : \kappa \\
& u_{1}, u_{2} \geq 0 . &
\end{array}
$$

The augmented Lagrangian function of the above problem is

$$
\begin{aligned}
& \mathscr{L}_{\mathscr{A}}\left(x, y, z, v, u_{1}, u_{2}, w ; \mu, p_{1}, p_{2}, v, \delta, \kappa\right) \\
= & \sum_{i=1}^{n}\left\|z_{i}\right\|+\sum_{i=1}^{n}\left\langle\mu_{i}, \nabla_{i} x-z_{i}\right\rangle+\frac{\beta_{1}}{2} \sum_{i=1}^{n}\left\|\nabla_{i} x-z_{i}\right\|^{2}
\end{aligned}
$$




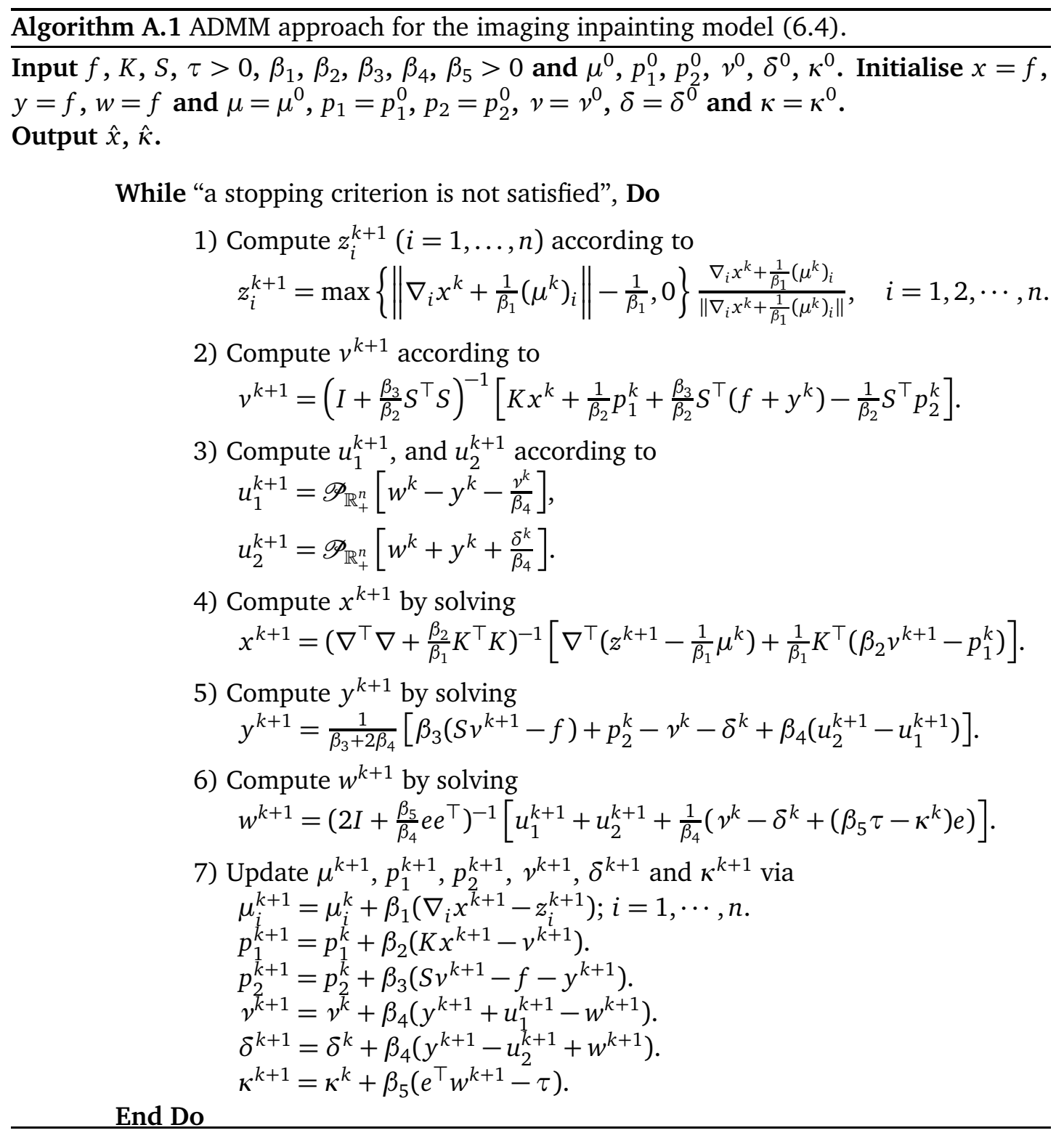

$$
\begin{aligned}
& +\left\langle p_{1}, K x-v\right\rangle+\frac{\beta_{2}}{2}\|K x-v\|^{2}+\left\langle p_{2}, S v-f-y\right\rangle+\frac{\beta_{3}}{2}\|S v-f-y\|^{2} \\
& +\left\langle v, y+u_{1}-w\right\rangle+\frac{\beta_{4}}{2}\left\|y+u_{1}-w\right\|^{2}+\left\langle\delta, y-u_{2}+w\right\rangle+\frac{\beta_{4}}{2}\left\|y-u_{2}+w\right\|^{2} \\
& +\left\langle\kappa, e^{\top} w-\tau\right\rangle+\frac{\beta_{5}}{2}\left\|e^{\top} w-\tau\right\|^{2},
\end{aligned}
$$

where $\beta_{1}, \beta_{2}, \beta_{3}, \beta_{4}, \beta_{5}>0$. With $\mu=\left(\mu^{(1)} ; \mu^{(2)}\right) \in \mathbb{R}^{2 n}$ and $\mu_{i}=\left(\mu_{i}^{(1)}, \mu_{i}^{(2)}\right)(i=1, \cdots, n)$, the approach for the image inpainting model (6.4) is summarised in Algorithm A.1 above. 


\section{References}

[1] G. Aubert and P. Kornprobst, Mathematical Problems in Image Processing: Partial Differential Equations and the Calculus of Variations, Springer-Verlag (2006).

[2] S.D. Babacan, R. Molina and A.K. Katsaggelos, Parameter estimation in TV image restoration using variational distribution approximation, IEEE Trans. Image Process. 17, 326-339 (2008).

[3] S.D. Babacan, R. Molina and A.K. Katsaggelos, Variational Bayesian blind deconvolution using a total variation prior, IEEE Trans. Image Process. 18, 12-26 (2009).

[4] D.P. Bertsekas, Convex Analysis and Optimization, Athena Scientific (2003).

[5] A. Bjőrck, Numerical Methods for Least Squares Problems, SIAM (1996).

[6] A. Bovik, Handbook of Image and Video Processing, Academic Press (2000).

[7] S. Boyd, N. Parikh, E. Chu, B. Peleato and J. Eckstein, Distributed optimization and statistical learning via the alternating direction method of multipliers, Found. Trends Mach. Learning 3, $1-122$ (2010).

[8] M. Carlavan and L. Blanc-Feraud, Sparse Poisson noisy image deblurring, IEEE Trans. Image Process. 21, 1834-1846 (2012).

[9] J.F. Cai, R.H. Chan and M. Nikolova, Fast two-phase image deblurring under impulse noise, J. Math. Imaging Vis. 36, 46-53 (2010).

[10] R.H. Chan and M.K. Ng, Conjugate gradient method for Toeplitz systems, SIAM Rev. 38, 427-482 (1996).

[11] T. Chan and J. Shen, Mathematical models for local nontexture inpaintings, SIAM J. Appl. Math. 62, 1019-1043 (2002).

[12] T. Chan and J. Shen, Image Processing and Analysis: Variational, PDE, Wavelet, and Stochastic Methods, SIAM (2005).

[13] T. Chan, J. Shen and H. Zhou, Total variation wavelet inpainting, J. Math. Imaging Vis. 25, 107-125 (2006).

[14] R.H. Chan, J.F. Yang and X.M. Yuan, Alternating direction method for image inpainting in wavelet domains, SIAM J. Imaging Sci. 4, 807-826 (2011).

[15] S.Y. Chun, Y.K. Dewaraja and J.A. Fessler, Alternating direction method of multiplier for tomography with non-local regularisers, IEEE Trans. Med. Imaging 33, 1960-1968 (2014).

[16] Y.Q. Dong, R. Chan and S.F. Xu, A detection statistic for random-valued impulse noise, IEEE Trans. Image Process. 16, 1112-1120 (2007).

[17] H. Fu, M. Ng, M. Nikolova and J. Barlow, Efficient minimization methods of mixed $\ell_{2}-\ell_{1}$ and $\ell_{1}-\ell_{1}$ norms for image restoration, SIAM J. Sci. Comput. 27, 1881-1902 (2006).

[18] D. Gabay and B. Mercier, A dual algorithm for the solution of nonlinear variational problems via finite-element approximations, Comput. Math. Appl. 2, 17-40 (1976).

[19] N.P. Galatsanos and A.K. Katsaggelos, Methods for choosing the regularisation parameter and estimating the noise variance in image restoration and their relation, IEEE Trans. Image Process. 1, 322-336 (1992).

[20] G.H. Gloub, M. Heath and G. Wahba, Generalized cross-validation as method for choosing a good ridge parameter, Technometrics 2, 215-223 (1979).

[21] G. Gilboa and S. Osher, Nonlocal operators with applications to image processing, Multiscale Model. Sim. 7, 1005-1028 (2008).

[22] R. Glowinski and A. Marrocco, Sur l'approximation par éléments finis d'ordre un et la résolution par pénalisation-dualité d'une classe de problèmes de Dirichlet non linéaires, Revue Fr. Autom. Inform. Rech. Opér., Anal. Numér. 2, 41-76 (1975).

[23] R. Gonzalez and R. Woods, Digital Image Processing, Addison-Wesley (1992).

[24] P.C. Hansen, Analysis of discrete ill-posed problems by means of the L-curve, SIAM Rev. 34, 287301 (1992). 
[25] P.C. Hansen, M.E. Kilmer and R.H. Kjeldsen, Exploiting residual information in the parameter choice for discrete ill-posed problems, BIT Numer. Math. 46, 41-59 (2006).

[26] H. Hwang, R.A. Haddad, Adaptive median filters: New algorithms and results, IEEE Trans. Image. Process. 4, 499-502 (1995).

[27] J.F.C. Mota, J.M.F. Xavier, P.M.Q. Aguiar and M. Püschel, A proof of convergence for the alternating direction method of multipliers applied to polyhedral-constrained functions, ArXiv 1112.2295 (2011).

[28] J.T. Kent and M. Mohammadzadeh, Global optimization of the generalized cross-validation criterion, Stat. Comput. 10, 231-236 (2000).

[29] C. Lawson and R. Hanson, Solving Least Squares Problems, Classics Appl. Math. 15, PrenticeHall (1974).

[30] H. Liao, F. Li and M. Ng, Selection of regularisation parameter in total variation image restoration, J. Opt. Soc. Amer. A 26, 2311-2320 (2009).

[31] V.A. Morozov, Methods for Solving Incorrectly Posed Problems, Springer (1984).

[32] M.K. Ng, R.H. Chan and W.C. Tang, A fast algorithm for deblurring models with Neumann boundary conditions, SIAM J. Sci. Comput. 21, 851-866 (1999).

[33] M.K. Ng, P. Weiss and X.M. Yuan, Solving constrained total-variation image restoration and reconstruction problems via alternating direction methods, SIAM J. Sci. Comput. 32, 2710-2736 (2010).

[34] J.P. Oliveira, J.M. Bioucas-Dias and M.A.T. Figueiredo, Adaptive total variation image deblurring: A majorization-minimization approach, Signal Process. 89, 1683-1693 (2009).

[35] L. Rudin, S. Osher and E. Fatemi, Nonlinear total variation based noise removal algorithms, Phys. D 60, 259-268 (1992).

[36] B.W. Rust and D.P. OĹeary, Residual periodograms for choosing regularisation parameters for ill-posed problems, Inverse Probl. 24, 034005 (2008).

[37] S. Setzer, G. Steidl and T. Teuber, On vector and matrix median computation, J. Comput. Appl. Math. 236, 2200-2222 (2012).

[38] Y. Xiao, J. Yang and X. Yuan, Alternating algorithms for total variation image reconstruction from random projections, Inverse Probl. Imaging 6, 547-563 (2012).

[39] J. Yang, W. Yin, Y. Zhang and Y. Wang, A fast algorithm for edge-preserving variational multichannel image restoration, SIAM J. Imaging Sci. 2, 569-592 (2009).

[40] J. Yang, Y. Zhang and W. Yin, An efficient TVL1 algorithm for deblurring multichannel images corrupted by impulsive noise, SIAM J. Sci. Comput. 31, 2842-2865 (2009).

[41] T. Teuber, G. Steidl and R. Chan, Minimization and parameter estimation for seminorm regularisation models with I-divergence constraints, Inverse Probl. 29, 035007 (2013).

[42] C.R. Vogel and M.E. Oman, Iterative methods for total variation denoising, SIAM J. Sci. Comput. 17, 227-238 (1996).

[43] Y. Wang, J. Yang, W. Yin and Y. Zhang, A new alternating minimization algorithm for total variation image reconstruction, SIAM J. Imaging Sci. 1, 948-951 (2008).

[44] C. Wu, J. Zhang and X. Tai, Augmented Lagrangian method for total variation restoration with non-quadratic fidelity, Inverse Probl. Imaging 5, 237-261 (2011).

[45] P. Weiss, L. Blance-Feraud and G. Aubert, Efficient schemes for total variation minimization under constraints in image processing, SIAM J. Sci. Comput. 31, 2047-2080 (2009).

[46] P. Weiss, G. Aubert and L. Blanc-Féraud, Some applications of $\ell^{\infty}$-constraints in image processing, INRIA Research Report 6115 (2006).

[47] Y.W. Wen and R. Chan, Parameter selection for total variation based restoration using discrepancy principle, IEEE Trans. Image Process. 21, 1770-1781 (2012). 